\title{
Schizophrenia Associated with Epileptiform Discharges without Seizures Successfully Treated with Levetiracetam
}

\author{
Dominique Endres', Evgeniy Perlov', Bernd Feige', Dirk-Matthias Altenmüller2, \\ Nils Venhoff ${ }^{3}$ and Ludger Tebartz van Elst ${ }^{1 *}$
}

\begin{abstract}
'Section for Experimental Neuropsychiatry, Department of Psychiatry, Faculty of Medicine, University of Freiburg, Freiburg, Germany, ${ }^{2}$ Freiburg Epilepsy Center, Department of Neurosurgery, Faculty of Medicine, University of Freiburg, Freiburg, Germany, ${ }^{3}$ Department of Rheumatology and Clinical Immunology, Faculty of Medicine, University of Freiburg, Freiburg, Germany
\end{abstract}

Background: Schizophrenia-like disorders can be divided into endogenic or primary, idiopathic, polygenetic forms, and different secondary, organic subgroups [e.g., (para)epileptic, immunological, degenerative]. Epileptic and paraepileptic explanatory approaches have a long tradition due to the high rate of electroencephalography (EEG) alterations in patients with schizophrenia.

OPEN ACCESS

Edited by:

Karl Bechter,

University of Ulm, Germany

Reviewed by:

Sabina Berretta,

Harvard Medical School, USA

Mark Cunningham,

Newcastle University, UK

*Correspondence:

Ludger Tebartz van Elst tebartzvanelst@uniklinik-freiburg.de

\section{Specialty section: \\ This article was submitted to Schizophrenia, \\ a section of the journal \\ Frontiers in Psychiatry}

Received: 27 October 2016

Accepted: 17 January 2017

Published: 08 February 2017

Citation:

Endres D, Perlov E, Feige B, Altenmüller D-M, Venhoff $N$ and

Tebartz van Elst L (2017) Schizophrenia Associated with Epileptiform Discharges without Seizures Successfully Treated with Levetiracetam.

Front. Psychiatry 8:12. doi: 10.3389/fpsyt.2017.00012
Case presentation: We present the case of a 23-year-old female patient suffering, since the age of 14 years, from a fluctuating paranoid-hallucinatory syndrome with formal thought disorder, fear, delusions of persecution, auditory, visual, and tactile hallucinations, as well as negative and cognitive symptoms. Laboratory measurements showed increased titers of antinuclear antibodies (ANAs) in the context of ulcerative colitis. While there was no clear history or evidence of epileptic seizures, the EEG showed generalized $3 \mathrm{~Hz}$ polyspike wave complexes. Under treatment with levetiracetam, the symptoms disappeared and the patient was able to complete vocational training.

Conclusion: The schizophrenia-like symptoms associated with epileptiform discharges but not overt seizures and the good response to antiepileptic treatment could be interpreted in the context of a (para)epileptic pathomechanism. The EEG alterations might be due to a polygenetic effect due to different genes. Mild immunological mechanisms in the framework of ulcerative colitis and increased ANA titers might have supported the network instability. This case report illustrates (1) the importance of EEG screenings in schizophrenia, (2) a potential pathogenetic role of epileptiform discharges in a subgroup of patients with schizophrenia-like symptoms, and (3) that antiepileptic medication with levetiracetam could be a successful treatment alternative in schizophrenia-like disorders with EEG alterations.

Keywords: epilepsy, schizophrenia, epileptiform discharges, levetiracetam, paraepileptic, LANI hypothesis

Abbreviations: ACR, American College of Rheumatology; ANA, antinuclear antibody; CSF, cerebrospinal fluid; EEG, electroencephalography; FIRDA, frontal intermittent rhythmic delta activity; GABA, $\gamma$-aminobutyric acid; HV, hyperventilation; ICA, independent component analyses; IRDA; intermittent rhythmic delta activity; LANI, local area network inhibition; SLE, systemic lupus erythematosus; SV2A, synaptic vesicle glycoprotein $2 \mathrm{~A}$. 


\section{BACKGROUND}

Schizophrenia-like disorders are characterized by delusional perception and delusions of control, hallucinations (e.g., commenting or discussing voices), thought insertion or withdrawal, cognitive impairment, thought disorders, or social withdrawal. ${ }^{1}$ In addition to primary, endogenic or idiopathic, polygenetic forms, different secondary pathophysiological mechanisms [e.g., (para)epileptic, immunological, degenerative] can be assumed. Because of the high rates of electroencephalography (EEG) alterations, ranging from 7 to $60 \%$ in patients with schizophreniform syndromes, epileptic and paraepileptic explanatory approaches have a long tradition (1-3). In line with this assumption, we reported the first case of a young patient with a schizophrenialike disorder, generalized spike-and-slow-wave complexes without epileptic seizures but with remission under treatment with valproate $(4,5)$. Immunological reasons might be due to autoantibody-associated autoimmune encephalitis, cerebral vasculitis, or collagenosis [e.g., systemic lupus erythematosus (SLE)] (6). Immunological effects might lead to network instability and therefore cause (para)epileptic phenomena (7). The detection of a (para)epileptic or immunological mechanism opens new treatment perspectives, in that antiepileptics or immunomodulators may be helpful $(4,5,7-10)$.

\section{CASE PRESENTATION}

\section{Clinical Presentation}

We present the case of a 23-year-old female office clerk suffering from fluctuating paranoid-hallucinatory symptoms since the age

${ }^{1}$ http://apps.who.int/classifications/icd10/browse/2010/en\#/F20-F29. of 14 years (2007). Therefore, the diagnosis of paranoid schizophrenia was made by different psychiatrists. Although taking neuroleptics, in the course of the disease, the patient developed five episodes (for several weeks) with paranoid-hallucinatory exacerbation. In these episodes, the patient suffered from formal thought disorder, fear, delusions of persecution, auditory hallucinations with commenting, discussing, and commanding voices, visual hallucinations with seeing maggots in her room, and tactile hallucinations with the feeling of being touched from behind. In parallel to these exacerbations, the patient developed severe negative and cognitive symptoms including attention and memory deficits, fatigue, depressive mood, and sleep disturbances thus completing the psychopathological features of comprehensive schizophrenia. Neurological and medical examinations were normal.

\section{Family History}

There was a positive family history for unipolar depression, which was diagnosed earlier in two sisters, both parents, and both grandmothers. There was no history for schizophrenia-like psychopathology, bipolar disorder, or epilepsy.

\section{Somatic and Developmental History}

Symptoms started 6 weeks after pain of the large joints. Therefore, a rheumatological disease was discussed. During an external work-up of repeated diarrhea, a chronic inflammation gut disease (ulcerative colitis) was diagnosed in 2014 and treated with mesalazine. No birth complications or in utero abnormalities were remembered; the birth was performed by cesarean section. The early childhood development was normal. No febrile convulsions or inflammatory brain diseases were remembered. The patient suffered mild cerebral contusions at the age of 4 and 12 years.

\section{TABLE 1 | Diagnostic findings.}

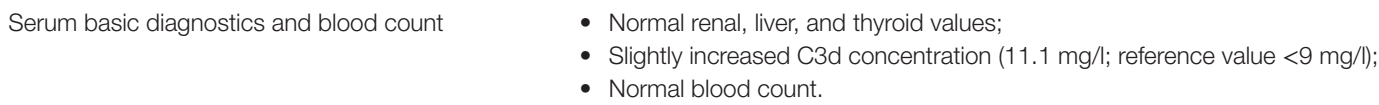

- Normal thyroid autoantibodies (against thyroglobulin, thyroid peroxidase, and thyroid-stimulating hormone);

- Rheumatological screening: increased antinuclear antibodies (titer: 1:400; reference value <1:50) without clear extractable nuclear antigens; the anti-nucleosome antibodies were weakly positive;

- No antibodies against intracellular onconeural antigens (Yo, Hu, CV2/CRMP5, Ri, Ma1/2, SOX1), or the intracellular synaptic antigens (GAD, amphiphysin).

Cerebrospinal fluid (CSF) analyses

- Normal white cell count, no blood-brain barrier dysfunction (normal protein concentration and albumin quotient);

- No CSF-specific oligoclonal bands, but a weak identical band in the CSF and serum;

- Antibodies against neuronal cell surface antigens [NMDAR, AMPA-R, GABA-B-R, VGKC complex (LGl1, Caspr2)] were negative.

Cerebral magnetic resonance imaging (1.5 T) • Normal brain findings;

- Additional examination findings included a benign lesion of the right frontoparietal skull without contrast enhancement (most likely equivalent with dermoid cysts; the criteria for monoclonal gammopathy of undetermined significance or multiple myeloma were not fulfilled).

Electroencephalography (during the first admission to our clinic in 2013, under the treatment with clozapine, aripiprazole, and citalopram)
- Frontal accentuated intermittent rhythmic delta activity (FIRDA) and generalized $3 \mathrm{~Hz}$ polyspike wave complexes. 

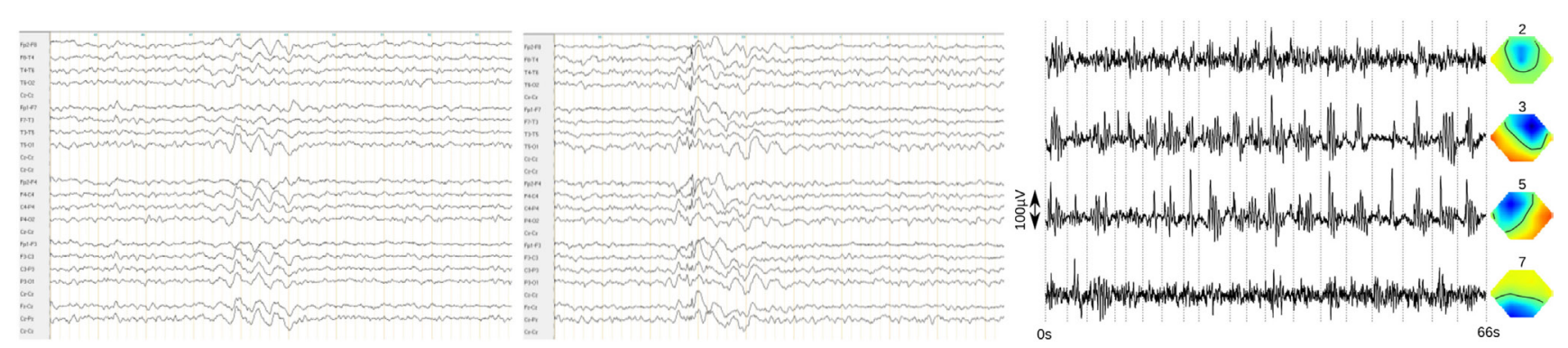

FIGURE 1 | Frontal accentuated intermittent rhythmic delta activity (FIRDA, left) and generalized $3 \mathrm{~Hz}$ polyspike wave complexes (middle) in the bipolar longitudinal rows $(7 \mu \mathrm{V} / \mathrm{mm}, 0.3 \mathrm{~s}, 70 \mathrm{~Hz})$. The findings of the independent component analysis are presented in the right picture [the following four relevant components were found, left: activity traces, with examples of atypical activity cut from the clinical electroencephalography and appended at the dotted lines. Right: topographies, nose upward, negative (direction opposite of activity trace) blue, positive (direction same as activity trace) red. Right (component 3) and left (component 5) frontal activity show most prominent $~ 2.6 \mathrm{~Hz}$ bursts. Higher frequency activity frontocentral (component 2) and occipital (alpha component 7) are partially related].

\section{Diagnostic Findings}

The diagnostic findings are summarized in Table 1. Taken together, the immunological alterations were compatible with the previously known ulcerative colitis (11). The electrophysiological findings (Figure 1) would be compatible with primary (idiopathic) generalized epilepsy; however, the history for epileptic seizures including absences and myoclonic jerks was negative.

\section{Differential Diagnosis}

The schizophreniform symptoms fulfilled the criteria of paranoidhallucinatory schizophrenia (see footnote 1 ). Therefore, the most important differential diagnoses would be schizophrenia plus coincidental epileptiform discharges. Although the findings of the investigation led us to our consideration of neuropsychiatric SLE, the American College of Rheumatology classification criteria for SLE were not fulfilled. ${ }^{2}$

\section{Therapy and Outcome}

External neuroleptic treatment with risperidone $(4 \mathrm{mg})$, amisulpride $(600 \mathrm{mg})$, perazine $(100 \mathrm{mg})$, aripiprazole $(15 \mathrm{mg})$, and clozapine $(275 \mathrm{mg}$ ) did not lead to long-term stabilization. Additional treatment with fluoxetine $(20 \mathrm{mg})$ and citalopram (20 mg) for affective, negative, and cognitive symptoms did not successfully improve these symptoms. During the first visit in our clinic, in 2013, we detected the abovementioned epileptiform discharges. Assuming a (para)epileptic pathomechanism, we added antiepileptic treatment with valproate $(1,500 \mathrm{mg})$ to the neuroleptic medication with clozapine and aripiprazole. At this point, the cognitive deficits improved significantly. Also, the EEG improved except for the (F)IRDAs. Another paranoid-hallucinatory episode in 2014 was treated successfully with a dose increase of clozapine and valproate. Because of a strong weight gain, the therapy with valproate was changed to topiramate $(200 \mathrm{mg})$ in 2014. Assuming a (para)epileptic pathomechanism, clozapine was reduced and stopped in 2014. Aripiprazole was reduced in

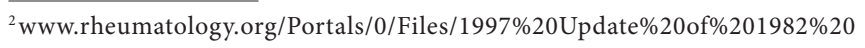
Revised.pdf.
January 2014 and stopped in 2015. Normal results were found in both the routine EEG and in the EEG after sleep deprivation (2014). In the further course, topiramate led to a severe loss of appetite and was therefore changed to levetiracetam (1,500 mg) in 2014. The mental condition stabilized with the short-term antiepileptic treatment with topiramate and the subsequent antiepileptic treatment with levetiracetam (since 2014, and since 2015 as monotherapy). There were no more paranoid-hallucinatory episodes, the negative symptoms declined, the patient became a mother (in the spring of 2016); she lived alone, took care of her daughter, and simultaneously finished her vocational training (in the summer of 2016). She was able to suspend the mesalazine therapy and therefore only took levetiracetam $(1,500 \mathrm{mg})$ at the time of stabilization.

\section{DISCUSSION}

We present the case of a patient with a schizophrenia-like disorder and, following our judgment, a (para)epileptic pathomechanism, because of the distinct epileptiform discharges without seizures and remission under the anticonvulsive treatment with topiramate and subsequently levetiracetam monotherapy.

\section{Reason and Potential Pathophysiology of Network Instability}

The EEG alterations might be due to a polygenetic effect caused by different genes (12). The immunological mechanisms in the framework of ulcerative colitis and increased antinuclear antibody titers might have supported the network instability by mild inflammatory processes $(7,13-15)$. Medication might also disclose underlying polygenetic or immunological network instability (16). The local area network inhibition (LANI) hypothesis might explain the causal relationship between epileptiform EEG discharges and schizophrenia-like symptoms. Excitatory network activity, as represented by the $3 \mathrm{~Hz}$ polyspike wave complexes, might lead to consecutive inhibitory processes in a physiological attempt of the central nervous system to stabilize the excitatoryinhibitory equilibrium of local cerebral networks. The repetitive 
excitatory activity, as documented by consecutive EEGs in our patient, could have exceeded a critical threshold, leading to the successive hyperinhibition of cerebral networks. Following the LANI hypothesis, the symptoms are due to the secondary induced processes of hyperinhibition (e.g., temporal hyperinhibition might have led to auditory hallucinations or memory deficits) $(2-5,17)$.

\section{Treatment Considerations}

Following the LANI hypothesis, the cognitive improvement after the addition of valproate to the neuroleptic treatment would be explained by the reduced epileptic activity and therefore the subsequent amelioration of inhibitory processes. In line with this assumption, comprehensive long-term stabilization was not achieved by several attempts of neuroleptic medication alone in spite of clear and very convincing effects of the treatment with clozapine in particular on positive symptoms. However, such a comprehensive improvement and even full remission was achieved with topiramate and later levetiracetam monotherapy. Thus, clozapine with its wellknown proconvulsive properties might well have counteracted inhibitory processes, while it is at the same time most likely unable to improve causative excitatory neuronal activity. By contrast, by reducing the epileptiform activity, topiramate and levitiracetam monotherapy might have resulted in a more causal and therefore more comprehensive improvement of relevant pathophysiology. Earlier, we published a case of a (para) epileptic schizophrenia-like disorder successfully treated with valproate (4). Valproate, and likewise lamotrigine, is already established as an augmentative treatment strategy in schizophrenia (18). One might hypothesize that patients with (para) epileptic pathomechanisms will benefit significantly more from antiepileptic treatment than other subgroups. To our knowledge, this is the first published case study that describes a patient with a schizophrenia-like disorder who was successfully treated with levetiracetam. Levetiracetam is rarely used off-label in psychiatry probably because of its potential side effects, such as agitation, aggression, fear, and psychosis (2). The advantage of levetiracetam is that it can be rapidly dosed up to effective concentrations. Therefore, on a single case basis, the working hypothesis of a (para)epileptic pathomechanism could be tested quickly. In comparison, valproate effects could be due to combined $\gamma$-aminobutyric acid (GABAergic) and antiglutamatergic effects, and lamotrigine effects might be due to potential antiglutamatergic effects. However, the mechanism of levetiracetam cannot be explained by such direct transmitter effects. The effects of levetiracetam seem to be associated with the binding of the synaptic vesicle glycoprotein 2A (SV2A) (2). SV2A can be found in presynaptic membranes; it controls the calcium-dependent exocytosis of different neurotransmitters into the synaptic gap $(19,20)$. Therefore, it might also influence GABAergic and glutamatergic transmission (21).

\section{Limitations}

Epileptiform discharges are found in less than $1 \%$ of healthy adults $(1,22-24)$ and as a rare consequence of clozapine treatment (16). Therefore, the EEG alterations could be interpreted either as an incidental finding in a patient with schizophrenia or as a clozapine side effect. However, the clinical course-with improvement under antiepileptic treatment in parallel with EEG normalization-speaks against the assumption. The pathophysiological processes might be explained by the LANI hypothesis; however, this is only an unproven, theoretical framework that needs further investigation.

\section{Conclusion}

This case report illustrates the idea of a possible (para)epileptic pathomechanism in a patient with a schizophrenia-like disorder. Regarding diagnostic procedure, our case shows the importance of EEG examinations in typical schizophrenia-like disorders. Regarding pathophysiology, the case illustrates a potential pathogenetic role of epileptiform discharges in a subgroup of patients with schizophrenia-like symptoms. Regarding treatment, the case demonstrates that anticonvulsive medication with levetiracetam and also topiramate or valproate could be a successful treatment alternative in schizophrenia with EEG alterations.

\section{ETHICS STATEMENT}

The patient has given her informed and written consent for this case report, including the presented images, to be published.

\section{AUTHOR CONTRIBUTIONS}

LTvE treated the patient. DE wrote the paper and performed the data collection. DE and LTvE performed the interpretation of the diagnostic findings and therapy effects. BF performed and interpreted the EEG analysis. NV performed and interpreted the immunological analyses. EP and D-MA reviewed the diagnostic results and contributed to the manuscript preparation. All the authors were significantly involved in the theoretical discussion and the preparation of the manuscript, and they read and approved the final version of the manuscript.

\section{REFERENCES}

1. Shelley BP, Trimble MR, Boutros NN. Electroencephalographic cerebral dysrhythmic abnormalities in the trinity of nonepileptic general population, neuropsychiatric, and neurobehavioral disorders. JNeuropsychiatry Clin Neurosci (2008) 20:7-22. doi:10.1176/appi.neuropsych.20.1.7

2. Tebartz van Elst L, Perlov E. Epilepsie und Psyche: Psychische Störungen bei Epilepsie - epileptische Phänomene in der Psychiatrie. 1st ed. Stuttgart: Kohlhammer (2013).

3. Endres D, Perlov E, Feige B, Fleck M, Bartels S, Altenmüller D, et al Electroencephalographic findings in schizophreniform and affective disorders. Int J Psychiatry Clin Pract (2016) 16:1-8. doi:10.1080/13651501.2016.1 181184

4. van Elst LT, Schulze-Bonhage A, Altenmüller D, Ebert D. Generalised spikeand-slow-wave complexes without seizures in schizophrenia. Br J Psychiatry (2011) 199:253-4. doi:10.1192/bjp.199.3.253a

5. Tebartz van Elst L, Krishnamoorthy ES, Schulze-Bonhage A, Altenmüller D, Richter H, Ebert D, et al. Local area network inhibition: a model of a 
potentially important paraepileptic pathomechanism in neuropsychiatric disorders. Epilepsy Behav (2011) 22:231-9. doi:10.1016/j.yebeh.2011. 06.016

6. Endres D, Perlov E, Dersch R, Baumgartner A, Hottenrott T, Berger B, et al. Evidence of cerebrospinal fluid abnormalities in patients with depressive syndromes. J Affect Disord (2016) 198:178-84. doi:10.1016/j.jad.2016.03.030

7. Tebartz van Elst L, Stich O, Endres D. Depressionen und Psychosen bei immunologischen Enzephalopathien. PSYCH up2date (2015) 9(05):265-80. doi:10.1055/s-0041-102941

8. van Elst LT, Klöppel S, Rauer S. Voltage-gated potassium channel/LGI1 antibody-associated encephalopathy may cause brief psychotic disorder. J Clin Psychiatry (2011) 72:722-3. doi:10.4088/JCP.10106510

9. Endres D, Perlov E, Stich O, Rauer S, Waldkircher Z, Lange T, et al. In vivo hypoglutamatergic state is associated with reduced cerebral glucose metabolism in anti-NMDA receptor encephalitis. BMC Psychiatry (2015) 15:186. doi:10.1186/s12888-015-0552-4

10. Endres D, Perlov E, Stich O, Tebartz van Elst L. Steroid responsive encephalopathy associated with autoimmune thyroiditis (SREAT) presenting as major depression. BMC Psychiatry (2016) 6:184. doi:10.1186/ s12888-016-0897-3

11. Barahona-Garrido J, Camacho-Escobedo J, García-Martínez C, Tocay H, Cabiedes J, Yamamoto-Furusho J. Antinuclear antibodies: a marker associated with steroid dependence in patients with ulcerative colitis. Inflamm Bowel Dis (2009) 15:1039-43. doi:10.1002/ibd.20852

12. Spillane J, Kullmann D, Hanna M. Genetic neurological channelopathies: molecular genetics and clinical phenotypes. JNeurol Neurosurg Psychiatry (2016) 87:37-48. doi:10.1136/jnnp-2015-311233

13. Hazouard E, Legras A, Diot E, Ferrandière M, Corcia P, Giniès G. [Cerebrospinal fluid complement and antinuclear antibodies in lupus meningoencephalitis]. Rev Neurol (Paris) (1998) 154:549-50.

14. Bonrath E, Rijcken E, Dziewas R, Vieth V, Bettenworth D, Senninger N, et al. [Cerebral vasculitis as rare extraintestinal manifestation in ulcerative colitis: review of the literature and case report]. Zentralbl Chir (2010) 135:350-3. doi:10.1055/s-0030-1247464

15. Bechter K. Updating the mild encephalitis hypothesis of schizophrenia. Prog Neuropsychopharmacol Biol Psychiatry (2013) 42:71-91. doi:10.1016/ j.pnpbp.2012.06.019

16. Treves I, Neufeld M. EEG abnormalities in clozapine-treated schizophrenic patients. Eur Neuropsychopharmacol (1996) 6:93-4. doi:10.1016/ 0924-977X(95)00057-V
17. Tebartz van Elst L, Fleck M, Bartels S, Altenmüller D, Riedel A, Bubl E, et al. Increased prevalence of intermittent rhythmic delta or theta activity (IRDA/IRTA) in the electroencephalogram (EEG) of patients with borderline personality disorder. Front Behav Neurosci (2016) 10:12. doi:10.3389/ fnbeh.2016.00012

18. Dold M, Leucht S. Pharmacotherapy of treatment-resistant schizophrenia: a clinical perspective. Evid Based Ment Health (2014) 17:33-7. doi:10.1136/ eb-2014-101813

19. Lynch B, Lambeng N, Nocka K, Kensel-Hammes P, Bajjalieh S, Matagne A, et al. The synaptic vesicle protein SV2A is the binding site for the antiepileptic drug levetiracetam. Proc Natl Acad Sci U S A (2004) 101:9861-6. doi:10.1073/ pnas. 0308208101

20. Zhang L, Li S, Li H, Zou X. Levetiracetam vs. brivaracetam for adults with refractory focal seizures: a meta-analysis and indirect comparison. Seizure (2016) 39:28-33. doi:10.1016/j.seizure.2016.05.004

21. Wood MD, Gillard M. Evidence for a differential interaction of brivaracetam and levetiracetam with the synaptic vesicle 2A protein. Epilepsia (2016). doi:10.1111/epi.13638 [Epub ahead of print].

22. Gregory RP, Oates T, Merry RT. Electroencephalogram epileptiform abnormalities in candidates for aircrew training. Electroencephalogr Clin Neurophysiol (1993) 86:75-7. doi:10.1016/0013-4694(93)90069-8

23. Bennett DR. Spike-wave complexes in "normal" flying personnel. Aerosp Med (1967) 38:1276-82.

24. Thorner MW. Procurement of electroencephalograph tracings in 1000 flying cadets for evaluating the Gibbs technique in relation to flying ability. USAD School of Aviation Medical Research Report No. 7-1. (1942).

Conflict of Interest Statement: DE, EP, and BF: none; D-MA: lecture fees from UCB Pharma; NV: advisory boards, lectures, research, or travel grants within the last 3 years: Janssen-Cilag, Roche, Novartis, AbbVie, GSK, and Medac. LTvE: lectures, work shops, or travel grants within the last 3 years: Eli Lilly, Medice, Shire, UCB, Servier, and Cyberonics.

Copyright $\odot 2017$ Endres, Perlov, Feige, Altenmüller, Venhoff and Tebartz van Elst. This is an open-access article distributed under the terms of the Creative Commons Attribution License (CC BY). The use, distribution or reproduction in other forums is permitted, provided the original author(s) or licensor are credited and that the original publication in this journal is cited, in accordance with accepted academic practice. No use, distribution or reproduction is permitted which does not comply with these terms. 